\title{
Interstitial lung disease-associated antisynthetase syndrome
}

\author{
I Hassan, MB BCh, MMed (Int), FCP (SA), Cert Pulm (SA) \\ Department of Intensive Care, School of Medicine, Sefako Makgatho Health Sciences University, Dr George Mukhari Academic Hospital, Ga-Rankuwa, \\ South Africa
}

Corresponding author: I Hassan (ismailhassan@mweb.co.za)

Interstitial lung disease (ILD) is one of the major extramuscular manifestations of polymyositis (PM) and dermatomyositis (DM). Presentation of PM and DM with ILD is not uncommon, but its clinical and radiological features can be similar to other conditions and can be challenging to diagnose. The presence of anti-aminoacyl tRNA synthetase antibodies in the presence of ILD is diagnostic of antisynthetase syndrome. A delayed diagnosis can be associated with the progression of pulmonary involvement and potentially increased morbidity. Timely diagnosis leads to appropriable lifesaving treatment. We report on a patient with chronic respiratory symptoms who had positive anti-Jo-1 antibodies and presented in respiratory failure, requiring non-invasive ventilation.

S Afr Respir J 2017;23(1):19-21. DOI:10.7196/SARJ.2017.v23i1.157

Antisynthetase syndrome (ASS) is a subtype of inflammatory myositis (polymyositis and dermatomyositis), and presents with a constellation of other clinical features. ${ }^{[1-3]}$ Acute dyspnoea in patients with the syndrome may be due to a spontaneous pneumomediastinum as a consequence of architectural distortion or vasculopathic lesions that result in alveolar and bronchial wall injury and subsequent air leaks. Other causes of acute dyspnoea or respiratory compromise may be pulmonary embolus, muscle weakness and pneumonia. ${ }^{[4,5]}$

Lung involvement may occur in the absence of muscular involvement. The anti-Jo-1 antibody was the first anti-ARS antibody to be discovered and is the most commonly reported autoantibody. ILD-associated anti-aminoacyl tRNA synthetase (ARS) antibodies are the most prevalent manifestation of ASS. ${ }^{[4-6]}$ We report a case of a patient with acute respiratory compromise in the setting of a positive anti-Jo-1 antibody, who was initially treated with non-invasive ventilation (NIV) and corticosteroids (CCSs), immune suppressants and a structured physiotherapy programme.

\section{Case report}

A 61-year-old female patient presented with sudden worsening of her chronic respiratory symptoms. She was misdiagnosed with asthma 6 years prior at another medical facility. Her medication included inhaled corticosteroids (ICSs), bronchodilators and methylxanthines. She was a never-smoker and had previously worked as a domestic helper. There was a history of arthralgia for which she used nonsteroidal anti-inflammatory drugs and paracetamol. She reported no other symptoms. She had late inspiratory crackles on examination of the chest. The rest of her examination was unremarkable.

She had respiratory failure upon admission and was started on NIV delivered by continuous positive airway pressure/pressure support (CPAP/PS), with a positive end expiratory pressure (PEEP) of $8 \mathrm{~cm}$ $\mathrm{H}_{2} \mathrm{O}$ and pressure support of $15 \mathrm{~cm} \mathrm{H}_{2} \mathrm{O}$. She was successfully weaned off NIV by day 10 to nasal prongs delivering $2 \mathrm{~L} / \mathrm{min}$.

Serological tests for myositis are shown in Table 1 . The patient's creatinine kinase was normal. There was no evidence of myositis. A diagnosis of ASS and ASS ILD was made. The lung function test (spirometry) depicted a mixed pattern with a restrictive and obstructive picture (Fig. 1), as seen in Table 2. The carbon monoxide diffusion was markedly reduced. The X-ray of the hands (Fig. 2), chest X-ray (CXR) (Fig. 3) and computed tomography (CT) scan of the chest (Fig. 4) are shown.

The patient had a favourable response to the NIV, pulse methylprednisolone $1 \mathrm{~g}$ daily for 3 days and antibiotics. After the acute episode, she was started on azathioprine and CCSs. During this time she underwent physiotherapy. At subsequent visits 4 weeks and 8 weeks later, she had an improvement in her 6 -minute walk test from $200 \mathrm{~m}$ to $400 \mathrm{~m}$ and her dyspnoea rating (assessed with the Borg scale) measured $4 / 10$.

\section{Discussion}

The ASS syndrome is diagnosed and characterised by the presence of aminoacyl tRNA antibodies (anti-ARS antibodies) accompanied by any one of the features listed in Table 3. The frequency of these associations is also shown in Table 3..$^{[2-4]}$ The ASS comprises various

Table 1. Serological tests for myositis

\begin{tabular}{ll}
\hline Myositis profile & Results for case patient \\
\hline Anti-Mi-2 & Negative \\
Anti-Ku & Negative \\
Anti-PM-Sc1100 & Negative \\
Anti-PM-Sc175 & Negative \\
Anti-Jo-1 & Positive \\
Anti-SRP & Negative \\
Anti-Pl-7 & Negative \\
Anti-Pl-12 & Negative \\
Anti-EJ & Negative \\
Anti-OJ & Negative \\
Anti-Ro-52 & Positive
\end{tabular}


Table 2. Spirometry results depicting a mixed pattern with a restrictive and obstructive picture

\begin{tabular}{|c|c|c|c|c|c|c|}
\hline Parameter & Pred & Pre & \%Pred & Post & \%Pred & $\% \operatorname{Diff}(2 / 1)$ \\
\hline VCmax (L) & 2.59 & 0.76 & 29 & 0.75 & 29 & -1 \\
\hline IC (L) & 1.85 & 0.31 & 17 & - & - & - \\
\hline ERV (L) & 0.75 & 0.45 & 60 & - & - & - \\
\hline FVC (L) & 2.50 & 0.52 & 21 & 0.75 & 30 & - \\
\hline FEV1 & 2.09 & 0.51 & 24 & - & - & 46 \\
\hline FEV1\%VCmax (\%) & 77.32 & 66.96 & 87 & - & - & - \\
\hline $\operatorname{PEF}(\mathrm{L} / \mathrm{s})$ & 5.72 & 3.48 & 61 & 2.75 & 48 & - \\
\hline FEF25 (L/s) & 5.14 & 3.34 & 67 & 2.75 & 54 & -21 \\
\hline FEF50 (L/s) & 3.48 & 2.03 & 58 & 2.67 & 77 & -20 \\
\hline FEF75 (L/s) & 1.22 & - & - & 1.57 & 128 & 31 \\
\hline F1V1 (L) & - & 0.70 & - & - & - & - \\
\hline PIF (L/s) & - & 1.60 & - & 1.88 & - & 18 \\
\hline
\end{tabular}

Table 3. Clinical findings in patients with ASS and positive tRNA synthetase antibodies

\begin{tabular}{ll}
\hline Feature & $\%$ \\
\hline Interstitial lung disease & 69 \\
Myositis & 90 \\
Symmetrical inflammatory arthritis & 5 \\
Fever, weight loss & 11 \\
Raynaud's phenomenon & 17 \\
Mechanic's hands & 17
\end{tabular}

combinations of ILD. This may present with usual interstitial pneumonia, nonspecific interstitial pneumonia, organising pneumonia or diffuse alveolar damage. The prevalence of these patterns varies, e.g. in a temporal sequence and some features may be more prominent than others. ASS syndrome affects women more than men, and the peak incidence is between the ages of 35 - 65 years. The mean age at presentation is 30 years. ${ }^{[1-5]}$

Respiratory symptoms may have an insidious onset or may be severe in the form of acute respiratory distress syndrome (ARDS). NIV instituted early may obviate the need for invasive mechanical ventilation. ${ }^{[6]}$

Although the patient tested negative in this case, the pl-7 and antipl-12 seem to be more frequently associated with the presence of fibrosis in the absence of myositis. ${ }^{[3,4,6]}$ Patients with new-onset and unclear ILD may not display signs of myositis or skin disease in the beginning. The lack of correlation between muscle and pulmonary symptoms often leads to a delayed diagnosis. The underlying CT pattern has no influence on disease progression or prognosis. ${ }^{[2,6]}$ The anti-RO52/SSA antibody (a marker for Sjögren syndrome) may occur together with the anti-Jo-1 antibody, as in the case described, and is associated with particularly severe ILD. ${ }^{[7]}$

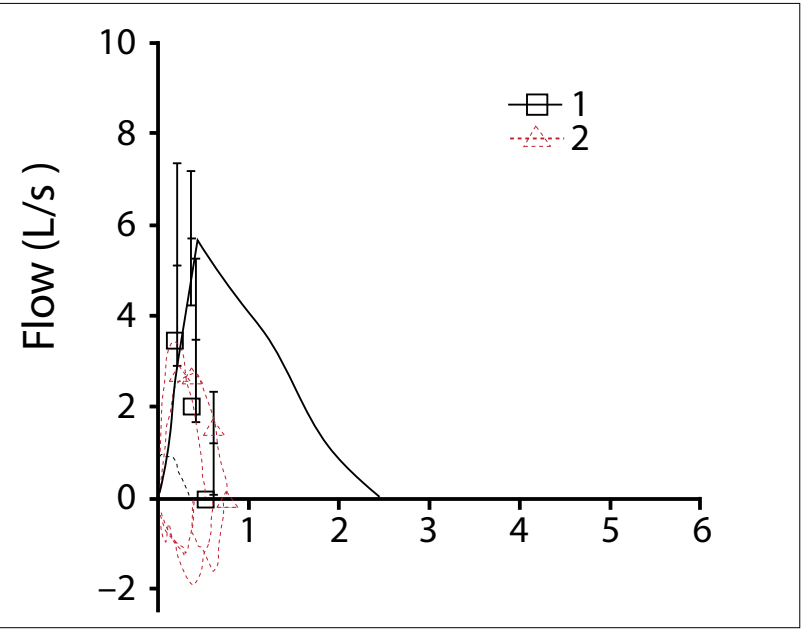

Fig. 1. Lung function test (spirometry) depicting a mixed pattern with a restrictive and obstructive picture.

A combination of drug treatment with a well-structured physiotherapy programme directed at resistance training and inspiratory muscle strength, over a period of several weeks, has an impact on overall wellbeing and symptom improvement. Physiotherapy can be beneficial in improving deconditioned muscles, but has no benefit in reversing fibrosis. ${ }^{[8,9]}$ Patients are able to do more with improved physical strength and with a greater understanding of the condition and how to manage it. ${ }^{[5,8,9]}$ This is evident from the improvement in the distance covered and dyspnoea rating during the 6-minute walk test.

Treatment is usually with CCSs and immunosuppressive agents. Mycophenolate mofetil has been used with success in some instances. Whether more aggressive therapy in $\mathrm{PM} / \mathrm{DM}$ patients presenting with ILD really leads to better outcomes is still an open debate. What is clear is that a high index of suspicion in this case led to a diagnosis..$^{[5,8,9]}$ The timely and appropriate treatment with NIV prevented the deterioration during the acute setting. ${ }^{[1,6,8,9]}$ 


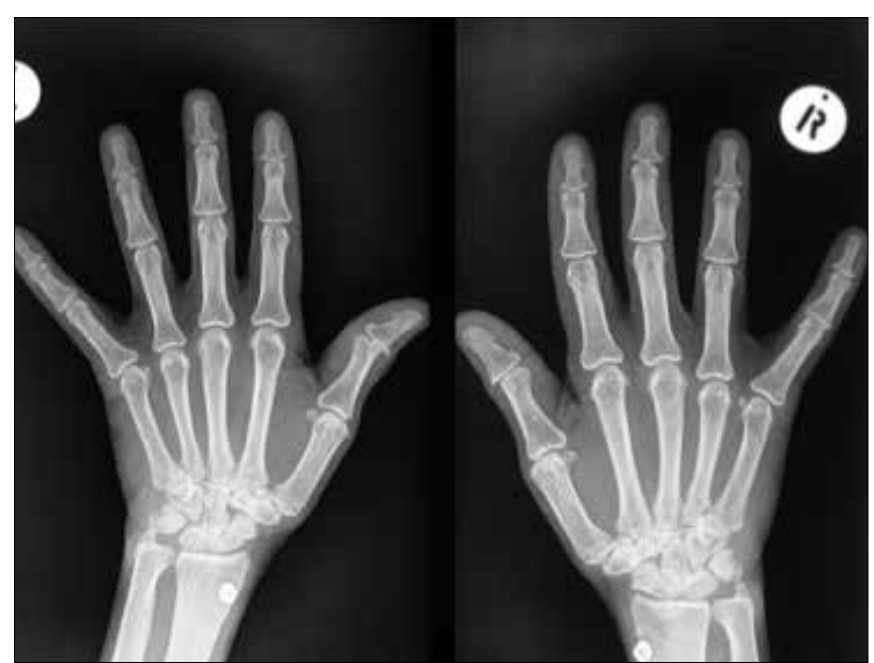

Fig. 2. X-ray of hands - no joint erosion seen.

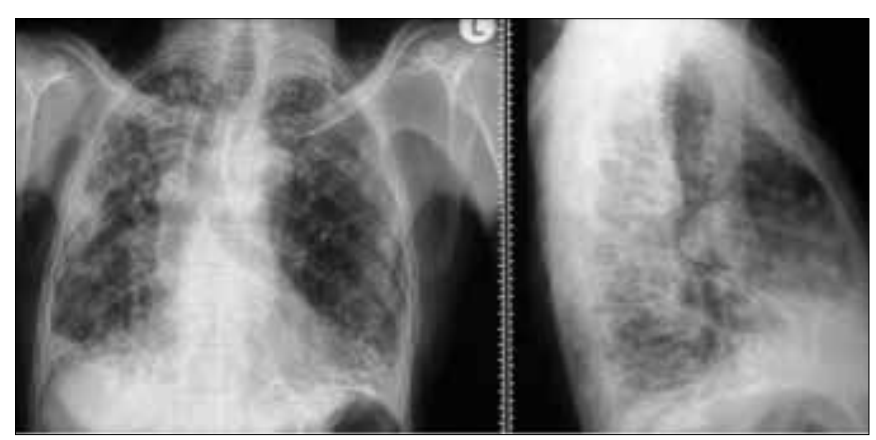

Fig. 3. CXR - coarse reticular pattern consistent with ILD.

We suggest that in the appropriate clinical context, patients with multisystem involvement presenting with arthralgias, Raynaud's phenomenon, myositis and lung involvement should be assessed for the ASS. A negative test for antinuclear antibodies (ANA) does not exclude the ASS syndrome. Frequent screening failures in the ANA test are not uncommon because the target antigen is mainly cytoplasmic in location. The routine screening for anti-Jo-1 antibody is recommended.

1. Douglas WW, Tazelaar HD, Hartman TE, et al. Polymyositis-dermatomyositisassociated interstitial lung disease. Am J Respir Crit Care Med 2001;164(7):1182-1185. http://dx.doi.org/10.1164/ajrccm.164.7.2103110

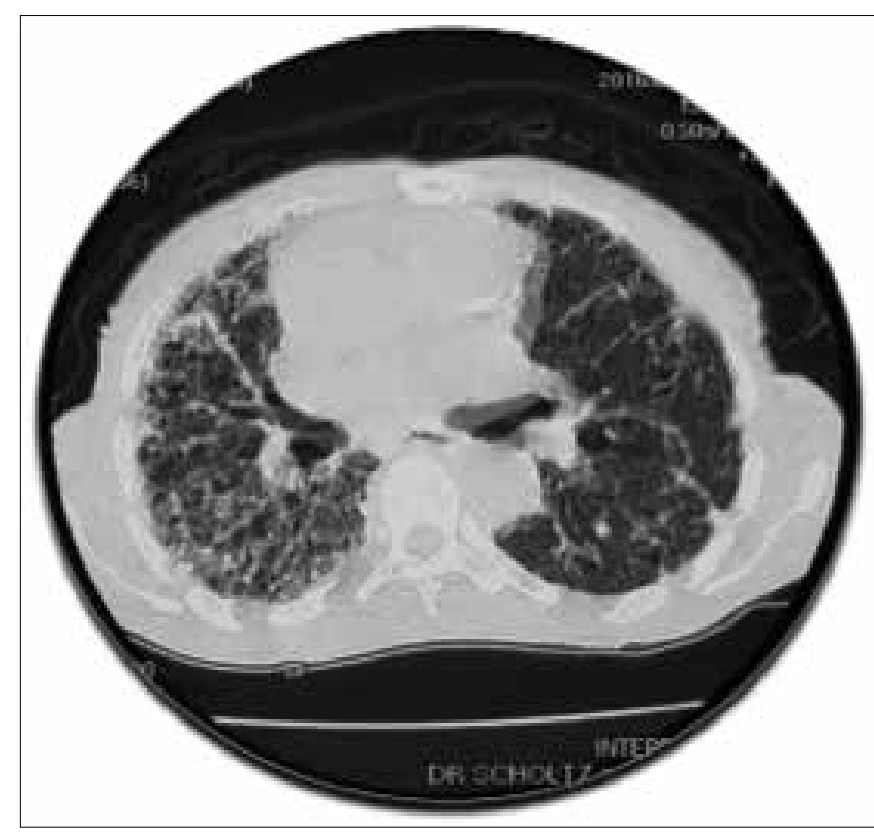

Fig. 4. CT scan showing reticulation, traction bronchiectasis fibrosis with some ground-glass appearance and architectural distortion.

2. La Corte R, Naco LM, Locaputo A, Dolzani F, Trotta F. In patients with antisynthetase syndrome the occurrence of anti-Ro/SSA antibodies causes a more severe interstitial lung disease. Autoimmunity 2006;39(3):249-253. https://doi. org/10.1080/08916930600623791

3. Viancsa A, Csipo I, Nemeth J, Devenyi K, Gergely L, Danko K. Characteristics of interstitial lung disease in SS-A positive inflammatory myopathy patients. Rheumatol Int 2009;29(9):989-994. https://doi.org/10.1007/s00296-009-0884-9

4. Won HJ, Soon Kim D, Keun Lee, et al. Two distinct clinical types of interstitial lung disease associated with polymyositis-dermatomyositis. Respir Med 2007;101(8):17611769. https://doi.org/10.1016/j.rmed.2007.02.017

5. Fischer A, du Bois R. Interstitial lung disease in connective tissue disorders. Lancet 2012;380(9842):689-698. http://dx.doi.org/10.1016/S0140-6736(12)61079-4

6. Park IN, Jegal Y, Kim DS, et al. Clinical course and lung function changer of idiopathic nonspecific interstitial pneumonia. Eur Respir J 2009;33(1):68-76. https://doi. org/10.1183/09031936.00158507

7. Kokosi M, Riemer EC, Highland KB. Pulmonary involvement in Sjögren syndrome. Clin Chest Med 2010;31(3):489-500. https://doi.org/10.1016/j.ccm.2010.05.007

8. Guglielmi S, Merz TM, Gugger M, Suter C, Nicod LP. Acute respiratory distress syndrome secondary to antisynthetase syndrome is reversible with tacrolimus. Eur Respir J 2008;31:213-217. https://doi.org/10.1183/09031936.00014707

9. Hervier B, Meyer A, Dieval C, et al. Pulmonary hypertension in antisynthetase syndrome: Prevalence, aetiology and survival. Eur Respir J 2013;42(5):1271-1282. https://doi.org/10.1183/09031936.00156312 